\title{
Near-Optimum Trellis-Coded DS/CDMA Multiuser Communications
}

\author{
Huang Lee, Kwang-Cheng Chen \\ Graduate Institute of Communication Engineering \\ National Taiwan University, Taipei, Taiwan, R.O.C. \\ hlee@ee.ntu.edu.tw, chenkc@ieee.org
}

\begin{abstract}
We propose two classes of sub-optimum receivers for coded direct-sequence code-division multiple-access (DS/CDMA) systems based on a generalized transmitter model. Compare to the optimum detector with complexity grows exponentially with the number of active user, the proposed detectors have linear complexity and near-optimum performance. The first class is to remove the multiple-access interference by modify the channel decoder. The second class is to separate the multiuser detection and channel decoder, and apply sub-optimum multiuser detector at the front stage. Because the proposed detectors are based on a generalized model, the detectors can be applied for many different transmitter schemes. Numerical results show that the proposed detectors are able to alleviate the near-far problem and the performance achieved by those detectors is near that by the optimum detector.
\end{abstract}

\section{INTRODUCTION}

In direct-sequence code-division multiple-access (DS/CDMA) systems, a number of users transmit information simultaneously and independently over a common medium. Each user is assigned a unique signature waveform such that the receiver can demodulate the desired information. The receiver receives a signal that is the sum of all users' signals plus noise. The bit-error-rate (BER) performance of the conventional detector suffers form multiple-access interference (MAI). In order to combat MAI, the optimum multiuser detector (MUD) is used to provide much better performance than the conventional detector [1]. However, the complexity of optimum MUD exponentially increases with the number of active users. To balance the trade-off between the performance and complexity, several sub-optimum detectors have been proposed, such as linear decorrelating detection [2], multistage detection [3], and others [1].

Channel coding is normally employed in communication systems to improve the performance. In particular, it was emphasized by Viterbi [4] that channel coding can increase bandwidth efficiency in spread-spectrum multiple-access (SSMA) systems. On the other hand, trellis-coded modulation (TCM) [5] is an efficient modulation scheme over additive white Gaussian noise (AWGN) channel. The rationals behind TCM is that coding and modulation should be designed jointly to maximize the normalized minimum Euclidean distance (free distance). The signal constellation, by applying set partitioning principle, can be expanded to provide redundancy for coding. In TCM, significant coding gain can be obtained without sacrificing data rate or bandwidth efficiency.
Some investigations have been done on coded DS/CDMA systems. Boudreau and Falconer [6] indicated that convolutionally coded DS/CDMA (CC-DS/CDMA) provides superior performance to trellis-coded DS/CDMA (TC-DS/CDMA) systems. Woerner and Stark [7] introduced a trellis-coded scheme over the expanded set, called bi-orthogonal signal set (BSS), of spreading sequences to increase the free distance. Choe and Georghiades [8] introduced the orthogonal plane sequence modulation (OPSM), which improves the power and bandwidth efficiency. Nevertheless, the aforementioned pioneer studies ignored the presence of MAI, which degrades the system performance. Fawer and Aazhang [9] used MUD to improve the performance based on the transmitter model in [7]. Giallorenzi and Wilson [10] [11] derived optimum and sub-optimum MUD for CC-DS/CDMA systems. In [12], we proposed a generalized model to investigate the trellis coding structure applied in DS/CDMA communications with MUD. We introduced the optimum detector for coded DS/CDMA systems and showed that significant coding gain can be achieved over uncoded systems by applying MUD with simple codes, and the performance of coded systems is degraded largely without MUD.

Nevertheless, the complexity of trellis structure in optimum receiver grows exponentially with the number of active users, information bits, and the states in each user's encoder. Because the complexity is prohibitively high, the optimum receiver for coded DS/CDMA systems is more unrealistic than that for uncoded systems. Hence, there is a need of suboptimum receivers, which are robust to near-far effect and have reasonable computational complexity. In this paper, based on the generalized model in [12], we propose sub-optimum receivers with linear complexity for the coded DS/CDMA systems and use the numerical analysis to demonstrate the system performance.

Typographic Convention: The superscripts $(\cdot)^{*},(\cdot)^{T}$, and $(\cdot)^{H}$ denote the complex conjugate, transpose, and Hermitian adjoint operator, respectively. A circumflex over any variable represents an estimate of that variable, such as $\widehat{x}$ for an estimate of $x$. The subspace spanned by the columns of a matrix $\mathbf{W}$ is represented with angle brackets around the symbol for the matrix, such as $\langle\mathbf{W}\rangle$. Given a set $\Omega,|\Omega|$ denotes the number of elements in the set $\Omega$. 


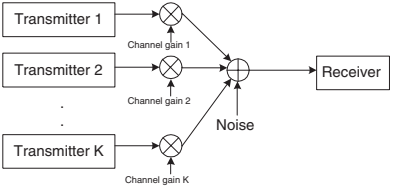

(a)

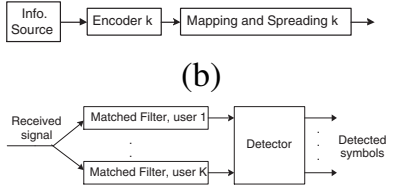

(c)
Fig. 1. (a) System block diagram. (b) Transmitter. (c) Receiver.

\section{SySTEM MODEL}

The general architecture of a coded DS/CDMA transceiver is illustrated in Fig. 1. Each user's transmitter comprises a binary memoryless source, a convolutional encoder, a memoryless mapping, and a spreading modulation. The detector jointly decodes the information bits and removes MAI. Consider a synchronous DS/CDMA system with $K$ users, we assume that (a) the encoders are identical with $\alpha$ input information bits, $\beta$ output coded bits, and constraint length $\nu$; (b) received amplitude and phase are perfectly estimated; (c) all users send the same number of total symbols.

We develop a generalized model, called multi-sequence, in the transmission of coded DS/CDMA systems. For user $k=$ $1, \cdots, K$, each user is assigned $D$ orthonormal spreading sequences (OSS), and the sequences are represented by $N_{c} \times 1$ vectors

$$
\underline{s}_{k, d} \triangleq \frac{1}{\sqrt{N_{c}}}\left[\begin{array}{ccc}
c_{k, d, 1} & \cdots & c_{k, d, N_{c}}
\end{array}\right]^{T}, d=1, \cdots, D
$$

where $c_{k, d, n} \in \mathcal{C}$ is the chip code with $\left|c_{k, d, n}\right|^{2}=1 ; N_{c}$ is the length of spreading sequences; and assume $N_{c}>$ $D K$. Moreover, each spreading sequence is multiplied by a coefficient $x_{k, d} \in \mathcal{R}$ or $\mathcal{C}$. For user $k$, the transmitted spreading sequence is given by

$$
\underline{s}_{k}=\sum_{d=1}^{D} x_{k, d} \underline{s}_{k, d} .
$$

In another point of view, the OSS of user $k$ can be considered composing a signal space $\left\langle\mathbf{W}_{k}\right\rangle$ where $\mathbf{W}_{k} \triangleq$ $\left[\begin{array}{llll}\underline{s}_{k, 1} & \underline{s}_{k, 2} & \cdots & \underline{s}_{k, D}\end{array}\right]_{N_{c} \times D}$. Naturally, (2) is doing the linear combination, and any signal point $\underline{s}_{k}$ used for transmission in this signal space $\left\langle\mathbf{W}_{k}\right\rangle$ can be arbitrarily designed by choosing different $x_{k, 1}, \cdots, x_{k, D}$. Because the encoder outputs $\beta$ bits each time, the transmitter needs to choose $2^{\beta}$ signal points in $\left\langle\mathbf{W}_{k}\right\rangle$ for transmission. Let the signal points used for transmission compose a set (signal constellation) $\Omega_{W, k} \in\left\langle\mathbf{W}_{k}\right\rangle$ with $\left|\Omega_{W, k}\right|=2^{\beta}$. The different signal constellation $\Omega_{W, k}$ can be described by defining the matrix

$$
\boldsymbol{\Phi}_{k} \triangleq\left[\begin{array}{cccc}
x_{k, 1}^{(1)} & x_{k, 1}^{(2)} & \cdots & x_{k, 1}^{\left(2^{\beta}\right)} \\
x_{k, 2}^{(1)} & x_{k, 2}^{(2)} & \cdots & x_{k, 2}^{\left(2^{\beta}\right)} \\
\vdots & \vdots & \ddots & \vdots \\
x_{k, D}^{(1)} & x_{k, D}^{(2)} & \cdots & x_{k, D}^{\left(2^{\beta}\right)}
\end{array}\right]_{D \times 2^{\beta}}
$$

where each column of $\boldsymbol{\Phi}_{k}$ specifies a signal point in $\Omega_{W, k}$, and $x_{k, d}^{(l)}$ stands for the $l$ th possible coefficient for spreading sequence $\underline{s}_{k, d}$.
While applying multi-sequence model, novel transmitters of coded DS/CDMA systems can be designed by adjusting the value $D$ and coefficient $x_{k, d}^{(l)}$ for different number of active users $K$ and length of spreading sequence $N_{c}$. For example, we construct a scheme by defining $x_{k, d}^{(l)} \in$ \{M-PSK signal constellation $\}$ for $D>1$ and $M^{D}=2^{\beta}$. When $M=2$, this scheme is equivalent to the multi-code scheme (MC) in multi-rate systems [13]. We denote this scheme with $D$ OSS as DMC-MPSK.

On the other hand, there are many previous coded DS/CDMA models can be regarded as a special case of multisequence model such as TC-DS/CDMA [6], BSS [7] and OPSM [8]. If the value $D$ is limited to be 1 and $x_{k, 1}^{(l)} \in$ \{PAM/PSK/QAM signal constellations $\}$ for $l=1, \cdots, 2^{\beta}$, the multi-sequence model degenerates into TC-DS/CDMA. In the BSS and OPSM model, each user chooses a spreading sequence between all possible transmitted OSS to transmit at each time. Each sequence contains a constellation that is limited to be BPSK and M-PSK for BSS and OPSM models, respectively. In fact, the BSS is a special case of OPSM. We therefore only introduce how to transform multi-sequence model into OPSM. By setting value $D$ equal to the number of possible OSS in OPSM with $M$-PSK $\left(M=\frac{2^{\beta}}{D}\right)$, multisequence mode can be degenerated into OPSM by letting

$$
x_{k, d}(l)=\left\{\begin{array}{ll}
m_{l-M(d-1)} & \text { if } d=\left\lceil\frac{l}{M}\right\rceil \\
0 & \text { otherwise }
\end{array},\right.
$$

$l=1, \cdots, 2^{\beta}, d=1, \cdots, D$ where $m_{p}, p=1, \cdots, M$ is the entries of $M$-PSK signal set.

The mathematical formulation of the transmitter is described as follows: At time index $i$, each user's source generates $\alpha$ information bits, and we denote user $k$ 's information bits by an $\alpha \times 1$ vector $\underline{b}_{k}[i] \triangleq\left[\begin{array}{llll}b_{k, 1}[i] & b_{k, 2}[i] & \cdots & b_{k, \alpha}[i]\end{array}\right]^{T}$ where $b_{k, m}[i] \in\{+1,-1\}$ for all $m$. For convenience, we define the $\alpha(2 I+1) \times 1$ information vector $\underline{b}_{k} \triangleq$ $\left[\begin{array}{llll}\underline{b}_{k}^{T}[-I] & \underline{b}_{k}^{T}[-I+1] & \cdots & \underline{b}_{k}^{T}[I]\end{array}\right]^{T}$ for user $k$; the information matrix $\mathbf{B} \triangleq\left[\begin{array}{llll}\underline{b}_{1} & \underline{b}_{2} & \cdots & \underline{b}_{K}\end{array}\right]_{\alpha(2 I+1) \times K}$; and the set $\Omega_{b, k}$ contains all possible vector $\underline{b}_{k}$.

The convolutionally coded symbols are generated by passing $\alpha$ information bits through a shift register consisting $2^{\nu}$ stages with modulo- 2 adders. The $k$ th user's $i$ th coded symbol is given by

$$
\begin{gathered}
u_{k}[i] \triangleq f\left(\underline{b}_{k}[i], \psi_{k}[i]\right) \in\left\{1, \cdots, 2^{\beta}\right\} \\
\psi_{k}[i+1] \triangleq g\left(\underline{b}_{k}[i], \psi_{k}[i]\right) .
\end{gathered}
$$

Function $f$ describes that the coded symbol depends not only on the corresponding vector $\underline{b}_{k}[i]$, but also on the state of encoder $\psi_{k}[i]$. Function $g$ describes the state evolution. The coded symbol $u_{k}[i]$ is mapped into the signal constellation $\Omega_{W, k}$ that consists $2^{\beta}$ distinct spreading sequences. The $i$ th transmitted waveform of user $k$ is

$$
s_{k}\left(t ; u_{k}[i]\right)=m\left(u_{k}[i]\right) \in \Omega_{W, k}
$$

where $m(\cdot)$ is the mapping function; $s_{k}\left(t ; u_{k}[i]\right) \triangleq$ $\sum_{d=1}^{D} x_{k, d}[i] s_{k, d}(t) ; s_{k, d}(t) \triangleq \frac{1}{\sqrt{N_{c}}} \sum_{n=1}^{N_{c}} c_{k, d, n} \Pi\left(t-n T_{c}\right)$; 
$x_{k, d}[i](d=1, \cdots, D)$ is the coefficients transmitted at time index $i$ and depends on $u_{k}[i] ; \Pi(\cdot)$ is a rectangular function of unit amplitude on the interval $\left[0, T_{c}\right)$; and $T_{c}$ is the chip duration.

The mapping (5) between coded symbols and spreading sequences is a key to combine the coding and spreading technique. For each user, since the performance is dominated by the free distance, while using Viterbi algorithm, coding and signal mapping should be designed jointly such that the distinct paths through the trellis are separated by larger distance. The Ungerboeck's set partitioning principle [5] is applied in the multi-sequence model to maximize the free distance. In order to apply Ungerboeck's principle, we can design the signal set $\Omega_{W, k}$ that is geometrically uniform [14] and use two-way geometrically uniform partitioning over $\Omega_{W, k}$. If the partitions admit binary isometric labelings, the coded bits can be mapped to transmitted signals by Ungerboeck's principle.

\section{Sub-optimum Detector}

Assuming an AWGN channel, the received signal that is the sum of all $K$ users' signals is given by

$$
r(t) \triangleq \sum_{k=1}^{K} \sum_{i=-I}^{I} a_{k} s_{k}\left(t-i T_{s} ; u_{k}[i]\right)+n_{w}(t)
$$

where $\left|a_{k}\right|$ and $\measuredangle a_{k}$ are the received amplitude and phase, respectively, of user $k ; T_{s}=N_{c} T_{c}$ is the symbol duration; and $n_{w}(t)$ is circularly symmetric white complex Gaussian noise with power spectral density $\sigma^{2} / 2=N_{0} / 4$ for real and imaginary part.

\section{A. Multistage Detector}

In order to combine the decision feedback detector and the channel decoder, we need to modify the branch metric that is a measure of distance among signal points in Viterbi algorithm. Before deriving the branch metric in sub-optimum detection, we need to obtain that in optimum detection.

Given (6), ML detector maximizes the function

$$
\begin{aligned}
& \operatorname{Re}\left\{2 \int_{-\infty}^{\infty} r^{*}(t) \sum_{k=1}^{K} \sum_{i=-I}^{I} a_{k} s_{k}\left(t-i T_{s} ; u_{k}[i]\right) d t\right\} \\
& -\int_{-\infty}^{\infty}\left|\sum_{k=1}^{K} \sum_{i=-I}^{I} a_{k} s_{k}\left(t-i T_{s} ; u_{k}[i]\right)\right|^{2} d t .
\end{aligned}
$$

After chip-matched filtering, which preserves the sufficient statistic, (7) is written in discrete-time form:

$$
\Lambda(\mathbf{B}) \triangleq \operatorname{Re}\left\{\sum_{i=-I}^{I}\left(2 \underline{y}^{H}[i] \mathbf{A} \underline{x}[i]-\underline{x}^{H}[i] \mathbf{A}^{H} \mathbf{R A} \underline{x}[i]\right)\right\}
$$

where $\underline{x}[i] \triangleq\left[\begin{array}{llll}x_{1,1}[i] & x_{1,2}[i] & \cdots & x_{K, D}[i]\end{array}\right]^{T}$ is a $K D \times 1$ vector; $\mathbf{S} \triangleq\left[\begin{array}{lll}\underline{s}_{1,1} & \cdots & \underline{s}_{K, D}\end{array}\right]_{N_{c} \times K D} ; \underline{y}[i] \triangleq$ $\left[\begin{array}{lll}y_{1,1}[i] & \cdots & y_{K, D}[i]\end{array}\right]^{T}$ is a $K D \times 1$ vector; $y_{k, d}[i] \triangleq$ $\int_{-\infty}^{\infty} r^{*}(t) s_{k, d}\left(t-i T_{s}\right) d t ; \mathbf{R} \triangleq \mathbf{S}^{H} \mathbf{S}$ is the $K D \times K D$ crosscorrelation matrix; and $\mathbf{A} \triangleq \operatorname{diag}\left\{a_{1}, \cdots, a_{K}\right\}$ is a $K D \times$ $K D$ diagonal matrix.
We show how to incorporate the channel decoders with sub-optimum MUD such as multistage detection [3]. At the receiver, users decode their received symbols separately and exchange detected bits information mutually. Each user removes MAI by adding others' detected bits information into the branch metric. Decision feedback detectors of this kind have to repeat decoding procedure iteratively.

Given $\Lambda(\mathbf{B})$ in (8), the multistage sub-optimum solution is now derived. Assume that the algorithm is in the $(m+1)$ th stage and is demodulating the sequence for the $k$ th user. While detecting $\underline{b}_{k}$ at $(m+1)$ th stage, we assume that the other users' signals are known and obtained from the previous stage. Denote $\underline{\underline{b}}_{k}^{(m)}$ as an estimate of vector $\underline{b}_{k}$ at the $m$ th stage. For $m \geq 1, \widehat{\widehat{b}}_{k}^{(m+1)}$ that is an estimate of the $k$ th user's information vector at the $(m+1)$ th stage is given by

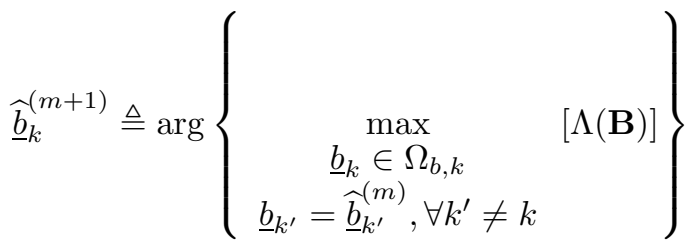

Equation (9) can be realized by Viterbi algorithm. The metric $M_{k}^{(m+1)}[i]$ for the $k$ th user at $(m+1)$ th stage is

$$
\begin{aligned}
& M_{k}^{(m+1)}[i] \\
& \triangleq \begin{cases}M_{k}^{(m+1)}[i-1]+\lambda^{(m)}\left(\underline{b}_{k}[i]\right), & i=-I, \cdots, I \\
0, & i<-I\end{cases}
\end{aligned}
$$

where

$$
\begin{aligned}
\lambda^{(m)}\left(\underline{b}_{k}[i]\right) & \triangleq \operatorname{Re}\left\{2 a_{k} \underline{y}_{k}^{H}[i] \underline{x}_{k}[i]-\left\|a_{k} \underline{x}_{k}[i]\right\|^{2}\right. \\
& \left.-2\left(a_{k} \underline{x}_{k}[i]\right)^{H} \xi_{k}^{H} \widetilde{\mathbf{A}}_{k} \widehat{\widehat{\widetilde{x}}}_{k}^{(m)}[i]\right\} ;
\end{aligned}
$$

$\underline{x}_{k}[i] \triangleq\left[\begin{array}{llll}x_{k, 1}[i] & x_{k, 2}[i] & \cdots & x_{k, D}[i]\end{array}\right]^{T}$ is a $D \times 1$ vector; $\underline{\widetilde{x}}_{k}[i]$ is a $(K-1) D \times 1$ vector that results by striking out all elements related to the user $k$ from the vector $\underline{x}[i]$; $\underline{y}_{k}[i] \triangleq\left[\begin{array}{llll}y_{k, 1}[i] & y_{k, 2}[i] & \cdots & y_{k, D}[i]\end{array}\right]^{T}$ is a $D \times 1$ vector; $\underline{\widehat{x}}_{k}^{(m)}[i]$ is an estimate of vector $\underline{\widetilde{x}}_{k}[i]$ at time index $i$ at $m$ th stage; $\xi_{k}$ is the $(K-1) D \times D$ matrix that is comprised of the columns related to the $k$ th user with the elements of the $k$ th user removed; $\widetilde{\mathbf{A}}_{k}$ is a $(K-1) D \times(K-1) D$ diagonal matrix that results by striking out all columns and rows related to the $k$ th user from the matrix $\mathbf{A}$.

In fact, the trellis structure in the proposed sub-optimum detector is the same as that in single user decoder, but the branch metric (11) that is a measure of distance among signal points has to be modified to adapt the multiuser case so that decoder can exchange information between users and thereby remove MAI. The last term in (11) is used to exchange MAI information between users. For the first stage, the last term in (11) can be dropped and the detector becomes conventional decoder without any MAI information or the decorrelating detector can be used as first stage [3] to improve the performance. 
For each user, the trellis structure has only $2^{\nu}$ states while each state has only $2^{\alpha}$ emanating and remerging branches. The complexity of $K$ users and $M$ stages detector is $M \cdot K \cdot 2^{\alpha+\nu}$ and grows linearly with the number of active users.

\section{B. Decorrelating Detector}

Another class of sub-optimum detectors is to separate the MUD and channel decoders. The sub-optimum MUD is performed first and delivers the output signals into each user's decoder separately. Decorrelating detector [2] is a linear MUD. Its complexity per demodulated symbol is linear in the number of active users. While implementing the decorrelator with channel decoder, the receiver can easily be decentralized. For each user, the output of the decorrelator is directly transferred into the decoder without any decision device so that it provides soft-decision information. The trellis structure in the decoder do not need to be modified and is just the same as that in single user case.

We derive the decorrelating detector for coded DS/CDMA systems. Given the vector form of the received signal:

$$
\underline{r}[i] \triangleq \sum_{k=1}^{K} a_{k} \sum_{d=1}^{D} x_{k, d}[i] \underline{s}_{k, d}+\underline{n}_{w}
$$

where $\underline{n}_{w}$ is complex Gaussian random vector while $E\left[\underline{n}_{w} \underline{n}_{w}^{\bar{H}}\right]=\sigma^{2} \mathbf{I}_{N_{c} \times N_{c}}$, and $E\left[\underline{n}_{w} \underline{n}_{w}^{T}\right]$ equals to a zero matrix. Recall the assumption that $a_{k}$ can be estimated perfectly. For user $k$, the signal $\underline{r}[i]$ is decorrelated by

$$
\underline{z}_{k}[i] \triangleq \frac{1}{a_{k}}\left(\mathbf{W}_{k}^{H} \mathbf{P}_{k} \mathbf{W}_{k}\right)^{-1} \mathbf{W}_{k}^{H} \mathbf{P}_{k} \underline{r}[i]
$$

where $\mathbf{P}_{k} \triangleq \mathbf{I}-\mathbf{T}_{k}\left(\mathbf{T}_{k}^{H} \mathbf{T}_{k}\right)^{-1} \mathbf{T}_{k}^{H}$ is a $N_{c} \times N_{c}$ matrix; and and $\mathbf{T}_{k}$ is the $N_{c} \times(K-1) D$ matrix that results by striking out all columns related to the user $k$ from the matrix $\mathbf{S}$.

Note that the noise term of user $k$ is enhanced by $\left(\mathbf{W}_{k}^{H} \mathbf{P}_{k} \mathbf{W}_{k}\right)^{-1} \mathbf{W}_{k}^{H} \mathbf{P}_{k}$, and its covariance matrix is $\sigma^{2}\left(\mathbf{W}_{k}^{H} \mathbf{P}_{k} \mathbf{W}_{k}\right)^{-1}$. When $D>1$, the covariance matrix is not a diagonal matrix in general. It means that the noise for user $k$ is not "white" anymore. This effect should be considered to improve the performance in the decoding algorithm as shown in (15). For user $k$, the information vector is decoded by taking Viterbi algorithm on

$$
\widehat{\underline{b}}_{k} \triangleq \arg \left\{\max _{\underline{b}_{k} \in \Omega_{b, k}}\left[\Lambda_{k}\left(\underline{b}_{k}\right)\right]\right\}
$$

$\Lambda_{k}\left(\underline{b}_{k}\right) \triangleq \sum_{i=-I}^{I}\left(\underline{z}_{k}[i]-\underline{x}_{k}[i]\right)^{H}\left(\mathbf{W}_{k}^{H} \mathbf{P}_{k} \mathbf{W}_{k}\right)\left(\underline{z}_{k}[i]-\underline{x}_{k}[i]\right)$

\section{Complexity Comparison}

Denote $E_{b}$ as bit energy. The comparison of optimum and sub-optimum detectors is shown in Table I. Because the decorrelator can be decentralized, users are able to decode their bits separately while applying decorrelating detector. The gap in complexity between the optimum detector and sub-optimum detectors are even larger in coded DS/CDMA systems than
TABLE I

COMPLEXITY AND BER COMPARISON FOR FIVE USERS. $\left(\frac{E_{b}}{N_{0}}=6 \mathrm{DB}\right)$

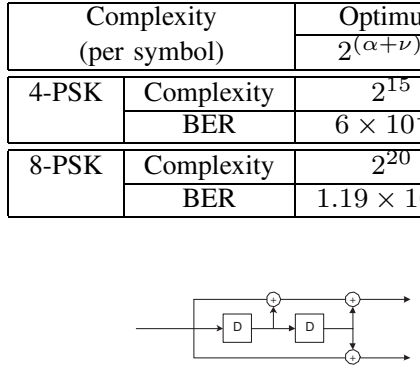

(a)

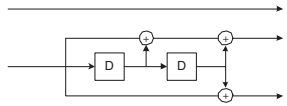

(b)
Fig. 2. Convolutional encoders.

that in uncoded systems. This is because that the number of encoder states and input bits also affect the complexity exponentially. The sub-optimum detectors are hence needed greatly to ensure practical implementation.

\section{Simulation Results}

In order to compare the uncoded and coded systems fairly, we have to maintain the bandwidth, coding rate and coding complexity the same in a simulation. The spreading sequences are composed of complex-valued Gold Code [15] and Walsh Code. In order to compare different situations, two different encoders are used as shown in Fig. 2. All simulations are in the multiple-access (five users) channel with AWGN, and all users have equal received energy except the near-far effect simulations. The length of spreading sequences $N_{c}$ is 32 .

We show that the performance is improved significantly by coding while applying sub-optimum detectors and degrades largely without MUD. Consider two different coding schemes, the simulation results of using encoder (a) and (b) are shown in Fig. 3 and Fig. 4, respectively. One-stage $(M=1)$ means that there is no feedback at the receiver, and each user do the decoding without eliminating any MAI (conventional detector). Increasing iteration number $M$ can improve the performance and makes the performance approach that of the optimum solution. The performance of the decorrelating detector is worse than that of the optimum detector because of the noise enhancement effect, which can be estimated by computing $\left(\mathbf{W}_{k}^{H} \mathbf{P}_{k} \mathbf{W}_{k}\right)$.

We investigate the near-far effect of the sub-optimum detectors as shown in Fig. 5. We use encoder (a) in this simulation, and the SNR of user 1 is fixed in $4 \mathrm{~dB}$. We show that the near-far problem is alleviated by the proposed detectors.

\section{CONCLUSION}

We proposed two different classes of sub-optimum detectors for coded DS/CDMA systems based on the multisequence model. For multistage detection, the branch metric was modified such that the channel decoders can exchange hard-decision information among users and thereby remove MAI. For decorrelating detection, the sub-optimum MUD 

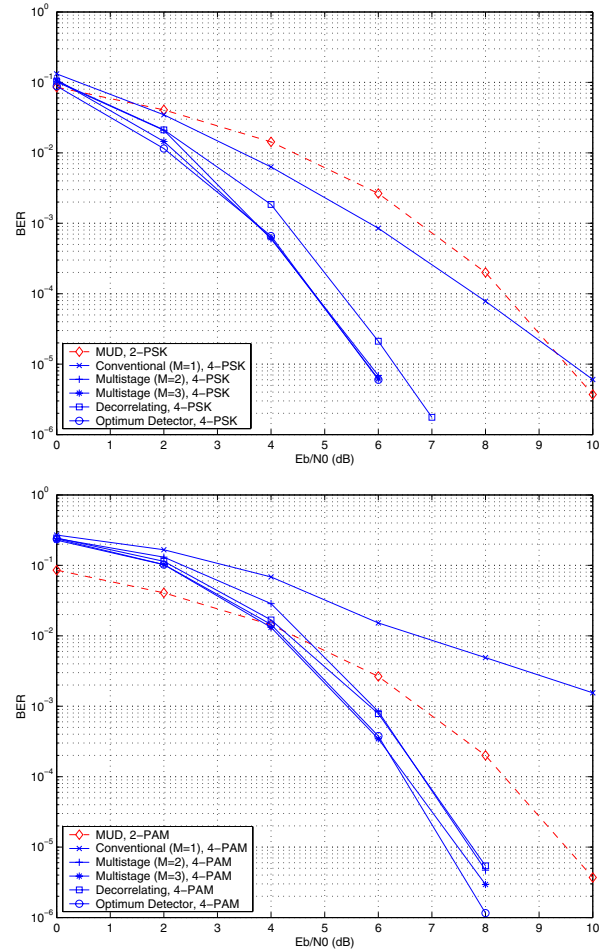

Fig. 3. BER versus SNR. Use encoder (a). Solid line represents coded system and dashed line stands for uncoded system.

provides soft-decision output to the channel decoders and the implement of the receiver can easily be decentralized. Since multi-sequence model is a generalized model for coded DS/CDMA systems, the proposed detectors can be applied to many schemes such as BSS, OPSM, and DMC-MPSK by adjusting the parameters of the detectors. The numerical results demonstrated that the proposed sub-optimum detectors have significant performance gain over the conventional decoder and have capability to alleviate the near-far problem.

\section{REFERENCES}

[1] S. Verdu, Multiuser Detection, 1st ed. Cambridge, U.K.: Cambridge Univ. Press, 1998.

[2] R. Lupas and S. Verdu, "Linear multiuser detector for synchronous codedivision multiple-access channels," IEEE Trans. Inform. Theory, vol. 35, no. 1, pp. 123-136, Jan. 1989.

[3] M. K. Varanasi and B. Aazhang, "Near-optimum detection in synchronous code-division multiple-access systems," IEEE Trans. Commun., vol. 39, no. 5, pp. 725-736, May 1991.

[4] A. J. Viterbi, "When not to spread spectrum - a sequel," IEEE Commun. Mag., vol. 23, no. 4, pp. 12-17, Apr. 1985.

[5] G. Ungerboeck, "Channel coding with multilevel/phase signals," IEEE Trans. Inform. Theory, vol. IT-28, no. 1, pp. 55-67, Jan. 1982.

[6] G. D. Boudreau, D. D. Falconer, and S. A. Mahmoud, "A comparison of trellis coded versus convolutionally coded spread-spectrum multipleaccess system," IEEE J. Select. Areas Commun., vol. 8, no. 4, pp. 628640, May 1990.

[7] B. D. Woerner and W. E. Stark, "Trellis-coded direct-sequence spreadspectrum communications," IEEE Trans. Commun., vol. 42, no. 12, pp. 3161-3170, Dec. 1994.

[8] S. Choe and C. N. Georghiades, "On the performance of a novel quasi-synchronous trellis-coded CDMA system," IEEE Trans. Commun., vol. 50, no. 12, pp. 1984-1993, Dec. 2002.
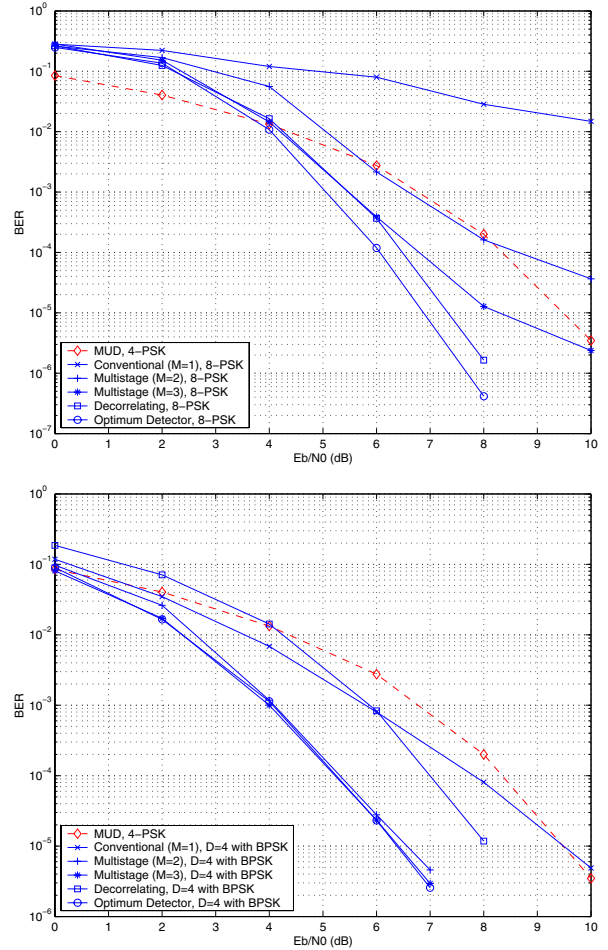

Fig. 4. BER versus SNR. Use encoder (b). Solid line represents coded system and dashed line stands for uncoded system.

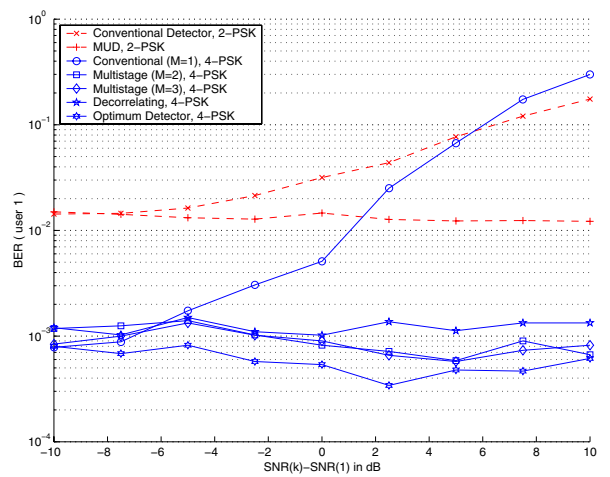

Fig. 5. BER for different near-far strength. $\mathrm{SNR}(1)=4 \mathrm{~dB}$. Solid line represents coded system and dashed line stands for uncoded system.

[9] U. Fawer and B. Aazhang, "Multiuser receivers for coded-division multiple-access systems with trellis-based modulation," IEEE J. Select Areas Commun., vol. 14, no. 8, pp. 1602-1609, Oct. 1996.

[10] T. R. Giallorenzi and S. G. Wilson, "Multiuser ML sequence estimator for convolutionally coded asynchronous DS-CDMA systems," IEEE Trans. Commun., vol. 44, no. 8, pp. 997-1008, Aug. 1996.

[11] _ - "Suboptimum multiuser receivers for convolutionally coded asynchronous DS-CDMA systems," IEEE Trans. Commun., vol. 44, no. 9, pp. 1183-1196, Sept. 1996

[12] H. Lee, "Trellis-coded DS/CDMA multiuser communications," Master's thesis, National Taiwan University, Taiwan, R.O.C., 2002.

[13] C.-L. I and R. D. Gitlin, "Multi-code CDMA wireless personal communications networks," in Proc. of IEEE ICC, vol. 2, Seattle, WA, June 1995, pp. $1060-1064$.

[14] J. G. D. Forney, "Geometrically uniform codes," IEEE Trans. Inform. Theory, vol. 37, no. 5, pp. 1241-1260, Sept. 1991.

[15] "3G TS 25.213 v4.2.0; spreading and modulation (FDD)," 3GPP Technical Specification, Dec. 2001. 